\title{
Prognosis of Interstitial Lung Disease Associated with Anti-Aminoacyl-tRNA Synthetase Antibodies: Look in the Middle
}

\author{
Vasilios Tzilas Argyris Tzouvelekis Evangelos Bouros Demosthenes Bouros \\ First Academic Department of Pneumonology, "Sotiria" Chest Diseases Hospital, Athens, Medical School, National \\ and Kapodistrian University of Athens, Athens, Greece
}

Antisynthetase syndrome is an autoimmune condition characterized by the presence of antibodies against aminoacyl-tRNA synthetase. The clinical signs and symptoms may vary depending on the presence of underlying myositis, polyarthritis, skin involvement (mechanic's hands), and Raynaud's phenomenon. Interestingly, patients with antisynthetase syndrome present a higher prevalence and increased severity of interstitial lung disease (ARS-ILD) comparing to patients with dermatomyositis and polymyositis. A lot of cases of ARS-ILD remain undiagnosed in clinical practice given the lack of awareness and also the fact that serology testing is usually restricted to anti Jo-1, even in cases with a high clinical suspicion [1-3].

In this issue of Respiration, Yamakawa et al. [4] provide valuable information regarding the natural history of ARS-ILD and relevant prognostic factors. Although the presence of antibodies against aminoacyl-tRNA synthetase was a prerequisite for inclusion in the study, not all patients had an established collagen tissue disease as 45 patients fulfilled the criteria of interstitial pneumonia with autoimmune features (IPAF) [5].This was a retrospective study that included 88 patients with more than 1 year data on pulmonary function tests (PFTs). The investigators studied the course of the disease based on the progression of PFTs. In particular, they studied 2 periods, an initial period corresponding to the first year and a long-term period between years 1 and 3 . In the initial period, patients were categorized as improved (increase $>10 \%$ in FVC and $>15 \%$ in DLco), worsened (decrease $>10 \%$ in FVC and $>15 \%$ in DLco), and stable (when not fulfilling neither of the above criteria). Regarding the initial period, 48 patients were improved, 21 patients were stable and 6 patients worsened. The long-term course of the disease was studied in the improved group that received anti-inflammatory therapy (corticosteroid alone or in combination with cyclosporine or tacrolimus) within 6 months after diagnosis based on the progression of PFTs between 1-year (initial data) and 3-year results. During the long-term period, data were available for 36 patients who were categorized as stable and unstable (patients who eventually deteriorated at 3 years despite ex-

\section{KARGER}

(c) 2018 S. Karger AG, Basel

E-Mail karger@karger.com

www.karger.com/res
Demosthenes Bouros, MD, PhD, FERS, FCCP, FAPSR

Academic Department of Pneumonology

Hospital for Diseases of the Chest "Sotiria", Medical School, University of Athen

Messogion Ave. 152, GR-11527 Athens (Greece)

E-Mail dbouros@med.uoa.gr 
hibiting an initial improvement at 1 year).The stable group included 24 patients and the unstable group $12 \mathrm{pa}$ tients. In the multivariate analysis there was no parameter that could be predictive of disease progression during the first year. For the long-term course of the disease, only middle lobe traction bronchiectasis was a significant prognosticator of deterioration despite initial improvement at 1 year (i.e., of the unstable group).The obvious limitations of this study are its retrospective and unicentric nature. Nevertheless, it offers significant and clinically valuable findings.

The prognosis of patients with ILD in the context of anti-synthetase syndrome is favorable and clearly better when compared to idiopathic pulmonary fibrosis (IPF) [6]. However, there is a subset of patients with progressive deterioration both in terms of PFTs and radiology. The radiographic aggravation is expressed as an increase in the overall disease extent usually accompanied by a transition to a more fibrotic phenotype. In a study by Debray et al. [7] in patients with anti-synthetase syndrome and ILD, most patients with increased disease extent over the course of time also showed progression or occurrence of honeycombing during follow-up. Early recognition of this subgroup is important as it will allow even closer follow-up and potentially more aggressive management. However, at the time being, there are no robust prognosticators. The current study by Yamakawa et al. [4] offers significant new insight. At first they showed that despite an initial improvement over the first year, one-third of patients eventually deteriorated after 3 years. The presence of middle lobe traction bronchiectasis was the sole prognostic factor in multivariate analysis. Thus, the clinician must remain vigilant despite an initial improvement phase, especially in patients with middle lobe traction bronchiectasis. It seems that the presence of fibrosis in the middle lobe is a surrogate marker of impending deterioration in the form of an ongoing fibrotic process. The clinical value of this finding remains to be prospectively validated. In the current study, there was no quantification regarding the extent of radiographic abnormalities, which is known to play an important prognostic role not only in fibrotic interstitial diseases in general but also in the context of antisynthetase syndrome [8]. It would be useful in an upcoming study to quantify the extent of inflammation and fibrosis according to the Goh score and the Kazerooni index and examine in multivariate analysis if the presence of middle lobe bronchiectasis still retains its prognostic significance.

Almost all of the patients exhibited an NSIP pattern on high-resolution computed tomography with (52\%) or without $(46.7 \%)$ features of organizing pneumonia (OP). Isolated OP pattern was rarely seen (1.3\%) and none of the patients had a usual interstitial pneumonia (UIP) pattern. Since there is no formal radiographic definition of OP pattern, NSIP with OP was identified when areas of consolidations superimposed on a background of radiographic NSIP. The findings are in agreement with the established knowledge that in ARS-ILD the most frequent radiology pattern is that of NSIP and that UIP is exceedingly rare. Characteristically, in ARS-ILD, there is a lower lobe predominance of radiographic abnormalities that hug or "pancake" the diaphragms $[9,10]$ and this was confirmed in the current study, as it was observed in the vast majority of patients $(97.3 \%)$.

Although not directly linked to the purpose of the study, a very interesting finding is that the second worst $\kappa$ value regarding interobserver agreement of high-resolution computed tomography findings was about determining the distribution of findings on the axial level, that is, peri-bronchovascular versus peripheral versus random. In the latest diagnostic criteria for IPF by Fleischner Society [11], determining the distribution of traction bronchiectasis as peripheral or not is of utmost importance with direct clinical implications. A peripheral distribution of traction bronchiolectasis with basilar predominance and in the absence of features suggestive of alternative diagnoses, is diagnostic of a probable UIP pattern allowing a diagnosis of IPF in the appropriate clinical background and obviating the need for tissue confirmation. The moderate $\kappa$ value even among experienced radiologists for determining the axial distribution of findings in this study highlights the practical difficulties and also the need for a universally accepted definition of peripheral versus central/peri-bronchovascular distribution [12].

According to the results from this study, ARS-ILD can have a variable course. The majority of patients have a favorable outcome after receiving anti-inflammatory treatment for 1 year showing stability or improvement. Despite the initial improvement, one-third of patients exhibit progressive worsening highlighting the need for increased vigilance and long-term follow-up. The presence of middle lobe traction bronchiolectasis was the sole parameter in multivariate analysis with prognostic significance over the long-term outcome. Prospective evaluation of this finding incorporating the extent of inflammation and fibrosis based on the Goh score and the Kazerooni index could help in establishing a robust radiographic prognosticator for patients with ARS-ILD. 


\section{References}

1 Papiris SA, Manali ED, Kolilekas L, Kagouridis K, Maniati M, Borie R, Pradere P, Crestani $B$, Bouros D: Investigation of lung involvement in connective tissue disorders. Respiration 2015;90:2-24.

2 Bouros D, Papiris S, Cottin V: Lung involvement in rheumatic disease: introduction Respiration 2015;90:1.

3 Papiris SA, Manali ED, Kolilekas L, Kagouridis K, Maniati M, Filippatos G, Bouros D: Acute respiratory events in connective tissue disorders. Respiration 2016;91:181-201.

4 Yamakawa $\mathrm{H}$, Hagiwara E, Kitamura H, Iwasawa T, Otoshi R, Aiko N, et al: Predictive factors for the long-term deterioration of pulmonary function in interstitial lung disease associated with anti-aminoacyl-tRNA synthetase antibodies. Respiration 2018; DOI: $10.1159 / 000488358$.
5 Fischer A, Antoniou KM, Brown KK, et al: "ERS/ATS task force on undifferentiated forms of CTD-ILD": an official European respiratory society/American thoracic society research statement: interstitial pneumonia with autoimmune features. Eur Respir J 2015; 46:976-987.

6 Tanizawa K, Handa T, Nakashima R, et al: The long-term outcome of interstitial lung disease with anti-aminoacyl-tRNA synthetase antibodies. Respir Med 2017;127:57-64.

7 Debray MP, Borie R, Revel MP, et al: Interstitial lung disease in anti-synthetase syndrome: initial and follow-up CT findings. Eur J Radiol 2015;84:516-523.

8 Rojas-Serrano J, Herrera-Bringas D, Mejía M, et al: Prognostic factors in a cohort of antisynthetase syndrome (ASS): serologic profile is associated with mortality in patients with interstitial lung disease (ILD). Clin Rheumatol 2015;34:1563-1569.
9 Waseda Y, Johkoh T, Egashira R, et al: Antisynthetase syndrome: pulmonary computed tomography findings of adult patients with antibodies to aminoacyl-tRNA synthetases. Eur J Radiol 2016;85:1421-1426.

10 Fischer A, Swigris JJ, du Bois RM, et al: Antisynthetase syndrome in ANA and anti-Jo-1 negative patients presenting with idiopathic interstitial pneumonia. Respir Med 2009;103: 1719-1724.

11 Lynch DA, Sverzellati N, Travis WD, et al: Diagnostic criteria for idiopathic pulmonary fibrosis: a Fleischner society white paper. Lancet Respir Med 2018;6:138-153.

12 Tzilas V, Valeyre D, Tzouvelekis A, Bouros D: Taking a giant step in the diagnosis of idiopathic pulmonary fibrosis. Lancet Respir Med 2018;6:82-84. 